\title{
Colistin: How should It Be Dosed for the Critically III?
}

\author{
Cornelia B. Landersdorfer, $\mathrm{PhD}^{1}$ Roger L. Nation, $\mathrm{PhD}^{1}$ \\ 1 Drug Delivery, Disposition and Dynamics, Monash Institute of \\ Pharmaceutical Sciences, Monash University, Melbourne, Australia \\ Semin Respir Crit Care Med 2015;36:126-135.

\begin{abstract}
Address for correspondence Roger L. Nation, PhD, Drug Delivery, Sciences, Monash University (Parkville Campus), 381 Royal Parade,

Cornelia B. Landersdorfer, PhD (e-mail: cornelia.landersdorfer@monash.edu).
\end{abstract} \\ Disposition and Dynamics, Monash Institute of Pharmaceutical \\ Parkville, Victoria 3052, Australia (e-mail: roger.nation@monash.edu).
}

\begin{abstract}
Colistin, an "old" polymyxin antibiotic, is increasingly being used as last-line treatment against infections caused by multidrug-resistant gram-negative bacteria. It is administered in patients, parenterally or by inhalation, as its inactive prodrug colistin methanesulfonate (CMS). Scientifically based recommendations on how to optimally dose colistin in critically ill patients have become available over the last decade and are extremely important as colistin has a narrow therapeutic window. A dosing algorithm has been developed to achieve desired plasma colistin concentrations in critically ill patients. This includes the necessary dose adjustments for patients with impaired kidney function and those on renal replacement therapy. Due to the slow conversion of CMS to colistin, a loading dose is needed to generate effective concentrations within a reasonable time period. Therapeutic drug monitoring is warranted, where available; because of the observed high interpatient variability in plasma colistin concentrations.

\section{Keywords}

- colistin

- critically ill

- polymyxins

- colistin methanesulfonate

- pharmacokinetics

- pharmacodynamics

- toxicodynamics

- optimized dosing Combination therapy should be considered when the infecting pathogen has a colistin minimum inhibitory concentration above $1 \mathrm{mg} / \mathrm{L}$, as increasing the dose may not be feasible due to the risk for nephrotoxicity. Inhalation of CMS achieves considerably higher colistin concentrations in lung fluids than is possible with intravenous administration, with negligible plasma exposure. Similarly, for central nervous system infections, dosing CMS directly into the cerebrospinal fluid generates significantly higher colistin concentrations at the infection site compared with what can be achieved with systemic administration. While questions remain to be addressed via ongoing research, this article reviews the significant advances that have been made toward optimizing the clinical use of colistin.
\end{abstract}

Colistin, together with polymyxin B, belongs to the group of polymyxin antibiotics which were discovered in the 1940s and introduced into patient care in 1959, but their clinical use was largely abandoned in the 1970s mainly due to concerns about their potential to cause nephrotoxicity. ${ }^{1}$ Over the last two decades, the emergence of gram-negative "superbugs" that are resistant to essentially all contemporary antibiotics and the lack of newly developed antibacterials have led to a resurgence in the use of the polymyxins. ${ }^{2-4}$ Parenteral products of both polymyxins exist; of the two polymyxins, colistin is more widely available around the world. For successful clinical use of any antibiotic, dosage regimens need to be optimized to maximize bacterial killing and minimize emergence of resistance and potential toxicity. This is important for any patient group but particularly so for the critically ill as they are most at risk for high morbidity and mortality. ${ }^{5}$ In addition, the above statement is especially true for colistin because, as reviewed below, it is an antibiotic with a narrow therapeutic window such that plasma concentrations that increase the risk for nephrotoxicity are not far above those required for the desired antibacterial effect.
Issue Theme Antimicrobial Resistance: Management of Superbugs; Guest Editor, David L. Paterson, MBBS, PhD, FRACP, FRCPA
Copyright (c) 2015 by Thieme Medical Publishers, Inc., 333 Seventh Avenue, New York, NY 10001, USA. Tel: +1(212) 584-4662. 
Colistin was never subjected to modern drug development and regulatory approval procedures which means that much of the information required to ensure its optimal use in patients has been unavailable. The recent researcher-led redevelopment of colistin has resulted in an improved understanding of its chemistry, parenteral formulations, pharmacokinetics (PK), pharmacodynamics (PD), and toxicodynamics (TD)., ${ }^{3,6,7}$ A good understanding of all of these key characteristics is required to optimize the clinical use of colistin and therefore these topics are briefly summarized below, followed by a review of the current knowledge on how to optimally dose colistin in critically ill patients.

\section{Chemistry, Units of Dosage, and Formulations}

Colistin is a cyclic polypeptide which is a cation at physiological $\mathrm{pH}$. Being a fermentation product it consists of several components, the major ones being colistin $\mathrm{A}$ and colistin B. ${ }^{8}$ It is administered parenterally (most often intravenously) and by inhalation as its inactive prodrug colistin methanesulfonate (CMS, also known as colistimethate) which has a lower potential for acute toxicity than colistin., 90 Conversion of CMS to colistin occurs in aqueous solutions both in vitro (e.g., water, bacterial growth media) and in vivo (e.g., plasma, urine).$^{9,11,12}$ Indeed, the conversion is a prerequisite for antibacterial activity to be unmasked.

CMS products for parenteral and inhalational use are standardized to the in vitro microbiological activity of colistin, but unfortunately labeling differs between geographic regions. ${ }^{2,13}$ Most notably in Europe, the United Kingdom, and India, CMS content and doses are expressed as the number of international units (IU). In North and South America, Southeast Asia, and Australia the amount in milligrams of colistin base activity (CBA) is used. A dose of one million IU corresponds to approximately $30 \mathrm{mg}$ of CBA (and approximately $80 \mathrm{mg}$ of the chemical CMS). These different conventions (especially expression of milligram amounts of two distinct entities) used for labeling and dosing have a great potential to cause confusion in clinical practice resulting in medication errors and serious consequences for patients. ${ }^{13,14}$ Awareness of the different terminology is required in clinical practice, especially when following recommendations in journal reports from different geographic regions. Articles for publication should use the recently recommended standardized terminology in expressing CMS doses. ${ }^{13}$

\section{Colistin Antibacterial Activity and Pharmacodynamics}

CMS is an inactive prodrug and therefore it is essential that colistin is used in in vitro studies, including measurement of minimum inhibitory concentration (MIC), that investigate activity against bacterial strains. ${ }^{9}$ Colistin is active against a range of gram-negative bacteria with most strains of Pseudomonas aeruginosa, Klebsiella pneumonia, and Acinetobacter baumannii being susceptible, even strains that are multiresistant to other antibiotics. ${ }^{15-18}$ The current susceptibility breakpoints for colistin are $\leq 2 \mathrm{mg} / \mathrm{L}$ for A. baumannii and Enterobacteriaceae, and $\leq 2 \mathrm{mg} / \mathrm{L}$ or $\leq 4 \mathrm{mg} /$ L for $P$. aeruginosa. ${ }^{19,20}$

Although the ultimate mechanism of bacterial killing is still not known, the initial bacterial target of colistin is the lipopolysaccharide (LPS) in the outer leaflet of the outer membrane of gram-negative bacteria. ${ }^{21}$ A key element in the interaction is electrostatic attraction of the positively charged amine groups of colistin with negatively charged phosphate and carboxylate groups on the lipid A and coreoligosaccharide of LPS. This electrostatic interaction enables interaction of the fatty acyl tail and other hydrophobic regions of the colistin molecule with hydrophobic domains of LPS. These electrostatic and hydrophobic interactions are believed to weaken the packing of adjacent lipid A fatty acyl chains causing substantial disruption and permeabilization of the outer membrane, including to colistin, a process termed "self-promoted uptake". 22 Subsequent steps in the killing action are not well defined and are subject to ongoing investigation. Clearly, the initial interaction between colistin and LPS is analogous to a "lock and key" arrangement and explains why colistin has very limited activity against grampositive bacteria. Not unexpectedly, most of the known mechanisms whereby gram-negative bacteria develop resistance to colistin involve either chemical modification of the phosphate groups of lipid A or elaboration of an outer membrane that lacks LPS, both being changes that attenuate the initial electrostatic interaction between colistin and the outer membrane. ${ }^{21,23}$

In vitro static and dynamic (the latter conducted in PK/PD models to mimic clinically relevant fluctuating concentrations in patients) concentration time-kill studies have demonstrated very rapid, concentration-dependent killing by colistin of multidrug-resistant $P$. aeruginosa, ${ }^{24-28}$ K. pneumonia $^{29,30}$ and $A$. baumannii. ${ }^{31-34}$ A common feature of such time-kill profiles is the regrowth of bacteria with enhanced resistance to colistin. This regrowth is often related to the phenomenon of colistin heteroresistance, which is the presence of a subpopulation of colistin-resistant bacteria in an isolate that would be considered susceptible on the basis of MIC. ${ }^{31}$ Following eradication of the predominant susceptible population, the colistin-resistant subpopulation undergoes unopposed amplification. The rate and extent of killing of $P$. aeruginosa in in vitro studies are considerably decreased at a high initial inoculum of $10^{8}$ or $10^{9}$ colony-forming units (CFU)/mL compared with a low initial inoculum of $10^{6} \mathrm{CFU} /$ $\mathrm{mL} .{ }^{35}$ At inocula of $10^{8}$ and $10^{9} \mathrm{CFU} / \mathrm{mL}$, killing of susceptible bacterial populations was approximately 6 -fold and 23 -fold slower, respectively, compared with an inoculum of $10^{6} \mathrm{CFU} /$ $\mathrm{mL}$. Clearly, the impact of the inoculum on the bactericidal activity of colistin requires further examination. However, the results of the study above ${ }^{35}$ imply that high-inoculum infections in patients may require more aggressive dosing. Colistin combination therapy should be considered for such infections because the risk of colistin-associated nephrotoxicity increases with plasma colistin concentrations above $\sim 2.5-3 \mathrm{mg} / \mathrm{L}$ as revealed by recent PK/TD analyses. ${ }^{36,37}$ However, as discussed in the next section, it is important to 
be aware of uncertainties that surround the role of colistin combination therapy.

Recent studies in an in vitro PK/PD infection model against $P$. aeruginosa ${ }^{26}$ and in the "gold standard" mouse thigh infection model against $P$. aeruginosa ${ }^{38}$ and $A$. baumannii ${ }^{39}$ have demonstrated that the PK/PD index that best correlates with the antibacterial activity of colistin is the ratio of the area under the concentration versus time curve to the MIC. These studies $^{26,38,39}$ suggest that it is important to achieve an average steady-state plasma colistin concentration of approximately $2 \mathrm{mg} / \mathrm{L}$ for isolates with MICs $\leq 1 \mathrm{mg} / \mathrm{L}$. This finding together with the relationship between plasma colistin concentration and risk of nephrotoxicity, ${ }^{36,37}$ as discussed above, indicates that colistin is an antibiotic with a narrow therapeutic window.

\section{Activity of Colistin in Combination with Other Antibiotics}

Studies conducted in in vitro static and dynamic infection models using clinically relevant concentrations of colistin and various second antibiotics have provided evidence for increased bacterial killing and decreased emergence of resistance with the use of certain colistin combinations against $P$. aeruginosa, A. baumannii, and K. pneumoniae. ${ }^{27,28,30,34,40}$ Not unexpectedly, the relative value of a combination may vary from bacterial strain to strain. ${ }^{41}$ Arguably, the most commonly tested second antibiotic has been a member of the carbapenem class. A recent systematic review and meta-analysis of in vitro studies explored the relative activity of colistin versus colistin plus carbapenem combinations. ${ }^{42}$ In general, across several carbapenems and bacterial species, bactericidal effect was enhanced and resistance emergence suppressed by the combination relative to the use of colistin alone. Across all bacterial species, of the carbapenems examined doripenem most consistently achieved synergy with colistin. ${ }^{42}$

Notwithstanding the growing evidence from in vitro studies for a beneficial effect of colistin combinations, the situation remains unclear in regard to the role of colistin combinations in patients. Very recently, an analysis was conducted of all clinical studies (12 retrospective cohort studies or case series, 2 prospective observational studies, and 2 randomized controlled trials [RCTs]) which compared colistin monotherapy versus colistin-based combination therapy for the treatment of infections caused by carbapenemase-producing or carbapenem-resistant gram-negative bacteria. ${ }^{43} \mathrm{~A}$ requirement for inclusion in the analysis was that the original studies reported quantitatively on the association between the treatment regimen and all-cause mortality. The analysis revealed that there was no difference in mortality between colistin alone and colistin/carbapenem combination therapy in any of the individual studies or when they were pooled. Pooling the only two RCTs showed similar mortality for colistin monotherapy versus colistin/rifampicin combination therapy. However, the authors of the analysis indicated that numerous sources of bias in the original studies existed, including the following: the retrospective nature of most of the studies; differences between the monotherapy and combination groups in regard to the nature and severity of infection; small sample sizes; appropriateness of the initial empirical antibiotic treatment; and the inclusion in some studies of multiple noncolistin antibiotics in the combination group. ${ }^{43}$ Additional limitations include the following: the use of dosage regimens of colistin and/or the second antibiotic that were not optimized based upon PK/PD principles; lack of measurement of plasma concentrations of colistin in both groups to gauge the equivalence of exposure to colistin; failure to stratify outcomes based on the site and/or severity of illness; and, the administration of antibiotics other than the index second antibiotic to patients in both the so-called colistin "monotherapy" group and the combination group. Clearly, given ethical and practical considerations, it is much more difficult to study colistin combinations in patients in the absence of potentially confounding effects than it is in a preclinical model where much tighter control over the experimental conditions is possible. Well designed and adequately powered RCTs are needed to define the role of colistin combination therapy. Two such RCTs (see NCT01732250 and NCT01597973 at ClinicalTrials.gov) are currently underway to compare colistin/carbapenem combination therapy versus colistin monotherapy for invasive infections caused by carbapenem-resistant gram-negative bacteria.

\section{Pharmacokinetics of CMS and Formed Colistin: General Considerations}

To optimize dosing of CMS/colistin, a good understanding of the PK of CMS and colistin is essential. Considerable progress has been made in this field since the beginning of the redevelopment of colistin and many reports of preclinical and clinical studies are available. ${ }^{44-50}$ It is important to be aware that "old" PK data (certainly the information generated before the start of the 21st century) based on CMS/colistin concentrations determined by microbiological assays are invalid due to the ongoing conversion of CMS to colistin during the incubation period of the assay. ${ }^{6}$ Despite this, PK data based on these outdated and erroneous findings are still included in product information and package inserts. ${ }^{2,51}$ This review will only consider PK data determined by high-performance liquid chromatography or liquid chromatographytandem mass spectrometry methods that are capable of separately quantifying CMS and formed colistin in biological fluids.

The inactive prodrug (CMS) and formed colistin (the active antibacterial) have very different PK (- Fig. 1). ${ }^{2,52}$ CMS is eliminated mainly via the kidneys, by glomerular filtration and there may also be a component of tubular secretion. ${ }^{45}$ Because in a renally healthy individual the renal clearance of CMS is much greater than its conversion clearance to colistin, only approximately $20 \%$ (or less) of a CMS dose is converted in vivo to the active entity colistin. ${ }^{2,52}$ Not only is the extent of conversion very low, but also the rate of conversion is slow. $^{48,50}$ Thus, CMS is a highly inefficient prodrug, and the clinical consequences of these characteristics for therapeutic use in critically ill patients will be discussed in the following section. In contrast, renal excretion plays a minor role in the 


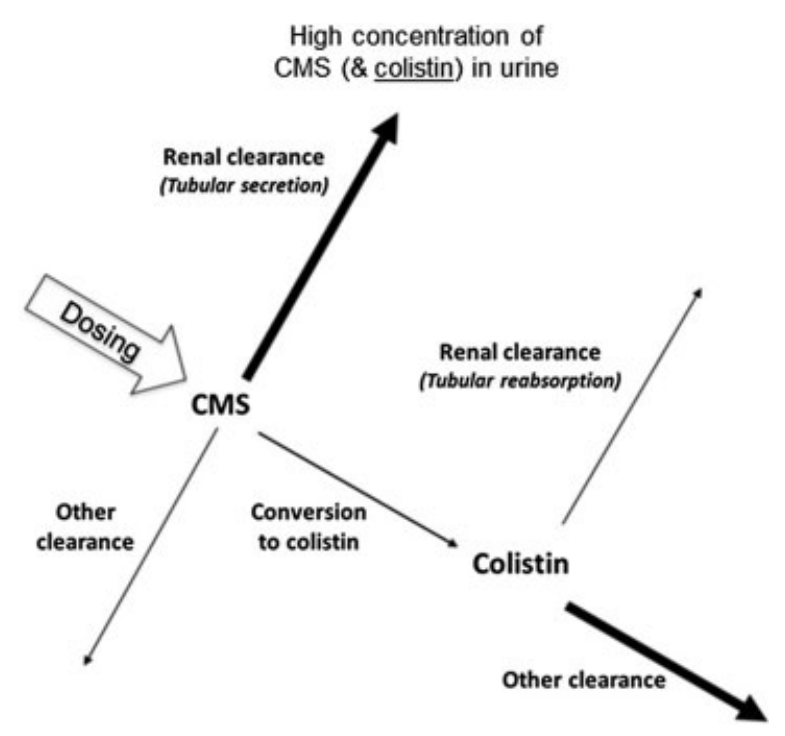

Fig. 1 Schematic diagram of the pharmacokinetic pathways for colistin methanesulfonate (CMS) and colistin. The thickness of the arrows indicates the relative magnitude of the respective clearance pathways when kidney function is normal. After administration of CMS, extensive renal excretion of the prodrug occurs with some of the excreted CMS converting to colistin within the urinary tract. Adapted with permission from Nation et al. ${ }^{52}$

overall elimination of formed colistin because following glomerular filtration colistin is subject to very extensive tubular reabsorption (-Fig. 1). 2,3,7,44 The reabsorptive trafficking of colistin through renal tubular cells is almost certainly linked to its propensity to cause nephrotoxicity.

\section{Pharmacokinetics of CMS and Formed Colistin in Critically III Patients: Implications for Dosing}

Initially, PK following intravenous administration of CMS will be considered. Subsequently, consideration will be given to administration of CMS directly to sites such as the lungs and the central nervous system. There has been only one brief report relating to three pediatric patients who ranged in age from 1.5 months to 14 years, ${ }^{53}$ and therefore the studies reviewed below relate to critically ill adult patients.

\section{Intravenous Administration of CMS}

The first report on the PK of intravenous CMS and the colistin formed from it in a critically ill patient, with plasma concentrations measured using specific chromatographic methods, was by Li et al. ${ }^{54}$ The patient was receiving continuous venovenous hemodiafiltration as part of management of multiorgan failure. Because the product information for CMS provided no information to guide dosage selection for such a patient, the patient was administered intravenously $2.5 \mathrm{mg}$ CBA per kg every 48 hour. This was a regimen that had been proposed in a review on antibiotic dosing in patients receiving continuous renal replacement therapy, although there was no supporting data for the suggested dosage regimen. ${ }^{55}$ The report of $\mathrm{Li}$ et $\mathrm{al}^{54}$ demonstrated that both
CMS and colistin were cleared by the renal replacement modality. As a consequence of the extracorporeal clearance and the inappropriately low daily dose of CMS, plasma concentrations of colistin were substantially lower than 1 $\mathrm{mg} / \mathrm{L}$, the MIC of the infecting organism, for almost $90 \%$ of the 48-hour dosage interval. Unfortunately, the patient did not survive. This case report sent a strong signal of the need for PK information to assist clinicians when selecting dosage regimens of CMS for various categories of critically ill patients.

Two subsequent small studies reported the steady-state plasma concentrations of formed colistin, but not CMS, following intravenous administration of CMS to critically ill patients, all of whom had creatinine clearance greater than about 50 $\mathrm{mL} / \mathrm{min} .{ }^{56,57}$ Patients were administered either 3 million IU (approximately $90 \mathrm{mg}$ CBA) every 8 hours ${ }^{56}$ or 2 million IU (approximately $60 \mathrm{mg}$ CBA) every 8 hours. ${ }^{57}$ Concern was expressed by the authors of both reports about the relatively low plasma colistin concentrations achieved in the patients. In these two studies, it was not possible to identify any patient factors that influenced the steady-state plasma colistin concentrations achieved. This was most likely due to the small number of patients ( $n=14$ and 13) included in the respective studies and the fact that all patients had creatinine clearance values greater than 46 and $96 \mathrm{~mL} / \mathrm{min}^{56,57}$

The PK of both CMS and formed colistin were investigated in two clinical studies involving a total of 28 critically ill patients who received intravenously 1 to 3 million IU (approximately 30-90 mg CBA) every 8 hours and most of whom had moderate-to-good renal function (creatinine clearance range $24-214 \mathrm{~mL} / \mathrm{min}$ ). ${ }^{48,58}$ These and other studies ${ }^{48,50,58}$ identified a significant problem that may arise if CMS regimens are not initiated with a loading dose. Because of the slow conversion of CMS to colistin mentioned above and the long half-life of formed colistin, in the absence of a loading dose of CMS plasma concentrations of colistin (the active antibacterial) rise slowly over the first 2 to 3 days of therapy. In the study of Plachouras et $\mathrm{al}^{48}$ a loading dose was not administered and plasma colistin concentrations were generally below $1 \mathrm{mg} / \mathrm{L}$ after the first dose (-Fig. 2, panel B). The long delay in achieving plasma colistin concentrations that are likely to be effective is of concern given the link between timely initiation of appropriate antibiotic therapy and clinical outcome in critically ill patients. ${ }^{59,60}$ Thus, a loading dose of CMS at the initiation of therapy is advised.

In the two studies mentioned above, ${ }^{48,58}$ plasma concentrations of CMS and colistin were also measured across a dosage interval at steady state. While accumulation had occurred relative to concentrations after the first dose, the plasma colistin concentrations across the dosage interval at steady state in several patients were less than $2 \mathrm{mg} / \mathrm{L}$ (-Fig. 2 , panel D). The authors expressed concern that the steady-state plasma concentrations of colistin were low in relation to current MIC breakpoints. ${ }^{48}$ The steady-state data along with those collected after the initial dose of CMS were pooled across the two studies and subjected to population PK analysis. $^{58}$ The clearance of the prodrug CMS was $13.1 \mathrm{~L} / \mathrm{h}$, its renal clearance was similar to creatinine clearance and the terminal half-life was 2.2 hours. The half-life of formed 

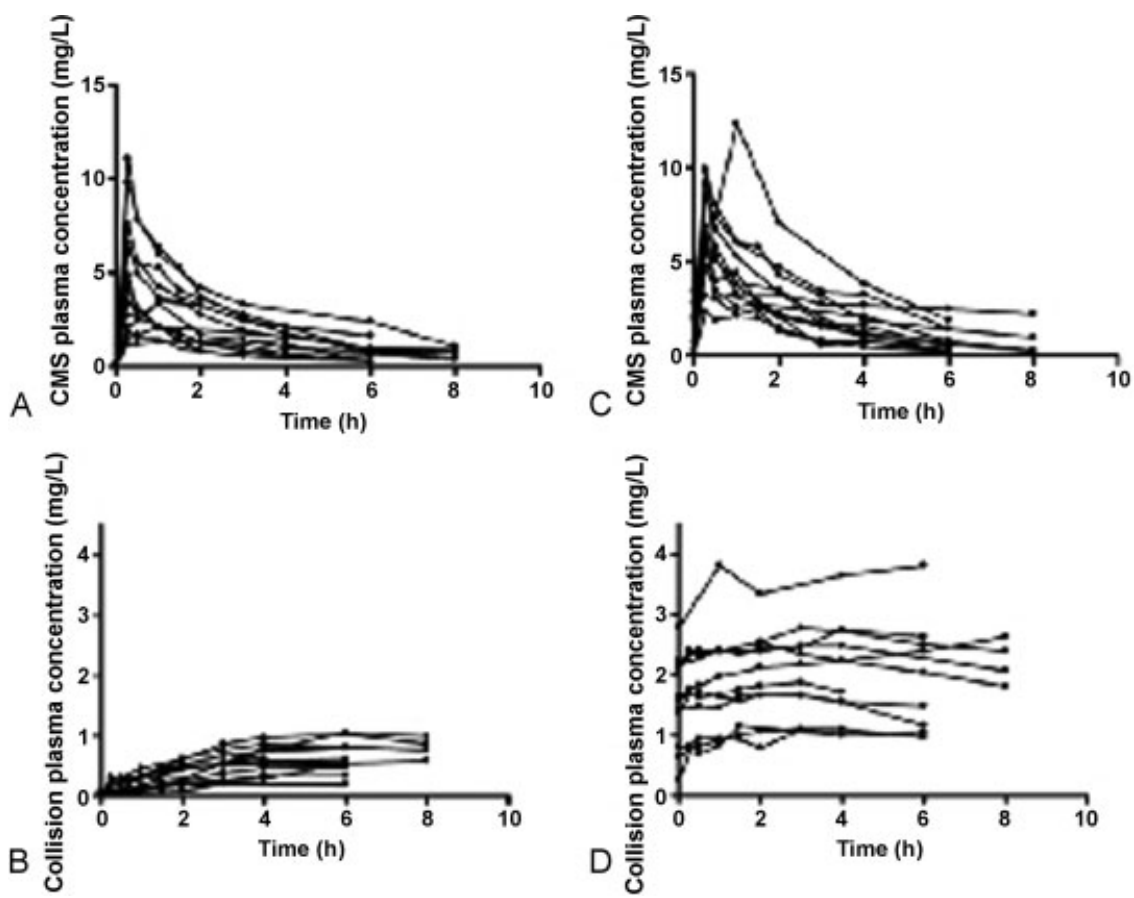

Fig. 2 Plasma concentrations of colistin methanesulfonate (CMS) (panels A and C) and formed colistin (panels B and D) in individual critically ill patients after the administration of the first dose of CMS (left panels) and the fourth dose of CMS (right panels). The dose of CMS was 3 million IU ( $\sim 90 \mathrm{mg}$ colistin base activity [CBA]) every 8 hours in all except one patient who received 2 million IU ( $\sim 60 \mathrm{mg}$ CBA) every 8 hours. Adapted with permission from Plachouras et al. ${ }^{48}$

colistin was considerably longer at 18.5 hours. A comprehensive search for patient covariates (e.g., body weight, renal function) that may influence the disposition of CMS and/or colistin was conducted by the authors. However, no covariates were identified, most likely because of the small sample size (total of 28 patients across the two studies) and only 3 of these patients had a creatinine clearance less than $50 \mathrm{~mL} / \mathrm{min}$.

Garonzik et $\mathrm{al}^{50}$ reported the results of the largest study thus far on the PK of CMS and colistin in critically ill patients. The study population was 105 patients, including 89 not on renal replacement who had a large range of renal function (creatinine clearance $3-169 \mathrm{~mL} /\left[\mathrm{min} \cdot 1.73 \mathrm{~m}^{2}\right]$ ), 12 on intermittent hemodialysis and 4 on continuous renal replacement therapy. The daily dose of intravenous CMS was at the discretion of the treating medical team. Across all patients the daily dose ranged from 75 to $410 \mathrm{mg}$ CBA (approximately 2.5-13.7 million IU) with a median of $200 \mathrm{mg}$ CBA (approximately 6.67 million $\mathrm{IU}$ ), and achieved an average plasma colistin concentration at steady state $\left(\mathrm{C}_{\mathrm{ss}, \text { avg }}\right)$ of 0.48 to $9.38 \mathrm{mg} / \mathrm{L}$ (median $2.36 \mathrm{mg} / \mathrm{L}$ )( - Fig. 3, panel B). That is, the approximately 5.5 -fold range in the daily dose of CMS resulted in approximately 20 -fold range in the $C_{s s, a v g}$ of colistin in plasma. Initial graphical analysis of the data suggested the likelihood that renal function, along with daily dose of CMS, was an important contributor to the wide range of plasma colistin concentrations observed (-Fig. 4). Also,
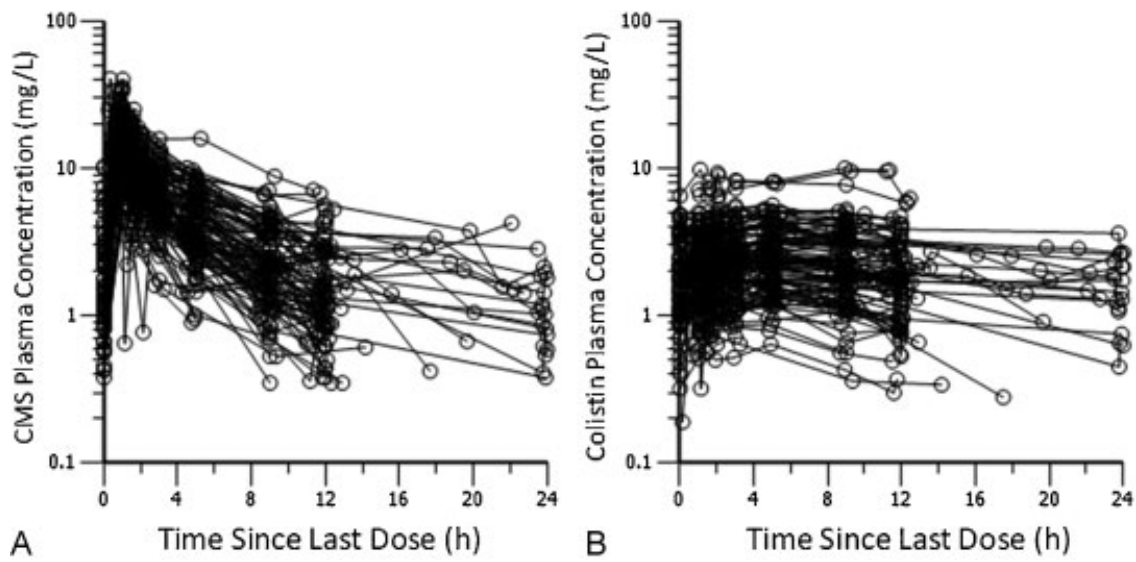

Fig. 3 Plasma concentration versus time profiles of the prodrug colistin methanesulfonate (CMS) (panel A) and formed colistin (panel B) across a dosage interval at steady state in 105 critically ill patients ( 89 not on renal replacement, 12 on intermittent hemodialysis, and 4 on continuous renal replacement therapy). Physician-selected CMS dosage intervals ranged from 8 to 24 hour and hence the interdosing blood sampling interval spanned the same range. Reproduced with permission from Garonzik et al. ${ }^{50}$ 

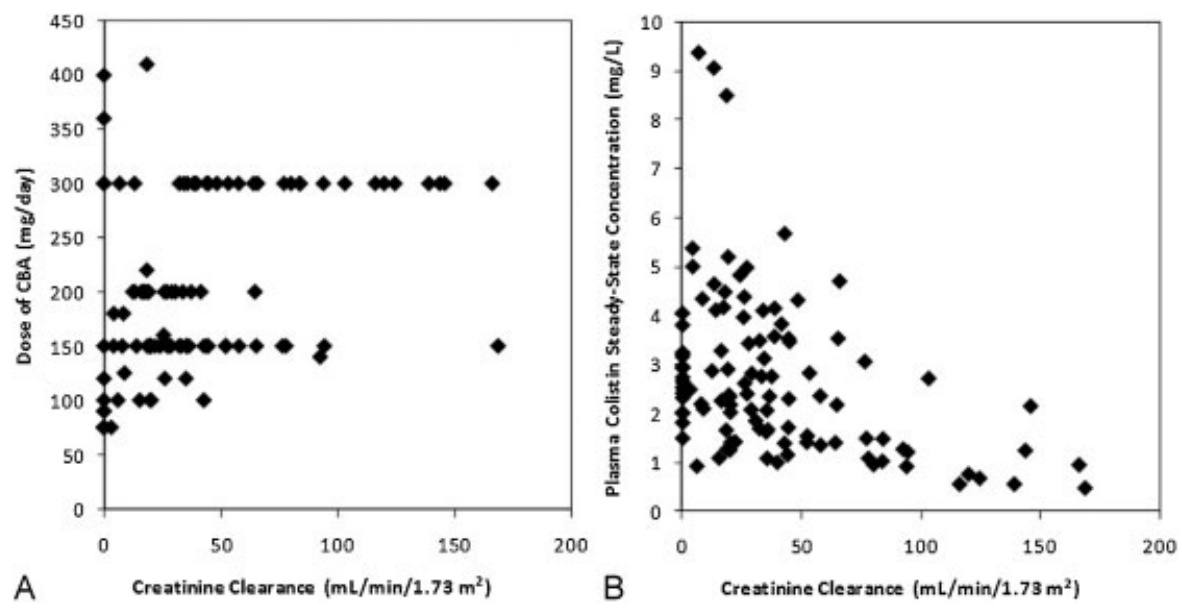

Fig. 4 Relationship of physician-selected daily dose of CMS (expressed as colistin base activity [CBA]) (panel A) and the resultant steady-state plasma colistin concentration (panel B) with creatinine clearance in 105 critically ill patients. Reproduced with permission from Garonzik et al. ${ }^{50}$

evident from these graphs was that administration of a daily dose of CMS at the upper limit of the currently approved dosage range (300 $\mathrm{mg} \mathrm{CBA} / \mathrm{d}$ ) was unable to reliably achieve a $\mathrm{C}_{\mathrm{ss}, \text { avg }}$ of colistin in plasma of $2 \mathrm{mg} / \mathrm{L}$. As noted above, this concentration may be considered as a reasonable target based upon translation of current evidence from PK/PD studies in animal infection models ${ }^{38,39}$ and given that PK/TD analyses indicate that the risk of nephrotoxicity in critically ill patients increases substantially as plasma colistin concentrations exceed approximately 2.5 to $3 \mathrm{mg} / \mathrm{L}^{36,37}$ Thus, in patients with relatively good renal function $(>\sim 80 \mathrm{~mL} / \mathrm{min})$, combination therapy should be considered, particularly if the MIC for the infecting organism is toward the upper end of the current breakpoint range. ${ }^{50}$

As the study of Garonzik et al ${ }^{50}$ comprised a large number of patients, including those with very low renal function, population PK analysis was able to identify creatinine clearance as a patient covariate that influenced the PK of both CMS and formed colistin. Because the prodrug (CMS) is predomi-

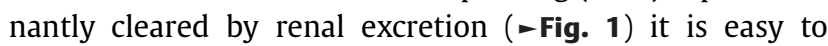
understand that its total body clearance declines with decreasing kidney function. It may be more difficult to understand the impact of declining renal function on the disposition of formed colistin given that renal excretion is a very small contributor to its overall elimination from the body. The explanation lies in the relatively complex interplay of the dispositions of CMS and colistin. In a patient with good kidney function, only a small fraction of each dose of CMS is converted to colistin ( $\mathbf{- F i g . 1}$ ). However, with declining renal function, a progressively larger fraction of each CMS dose is converted to the active antibacterial. Thus, the apparent clearance of colistin decreases in parallel with creatinine clearance. Not unexpectedly, creatinine clearance was the patient factor included in the algorithm developed by the authors to calculate the CMS daily maintenance dose needed to generate a desired target steady-state plasma concentration of formed colistin in a patient not receiving renal replacement therapy. ${ }^{50}$

Reflected by the data in - Fig. 4, at a given creatinine clearance there was a very large degree of interpatient variability (up to $\sim 10$-fold) in the apparent clearance of colistin and consequently in the CMS daily dose to achieve a desired steady-state plasma colistin concentration. The interpatient variability in the plasma colistin concentration achieved at a certain creatinine clearance and daily dose of CMS serves to complicate the clinical use of CMS, particularly since colistin has a narrow therapeutic window. Because of this wide interpatient variability in PK, clinicians are encouraged to use therapeutic drug monitoring (TDM) when available to assist in titration of the daily maintenance dose of CMS to achieve the desired steady-state plasma concentration of colistin. $^{52}$

Of the 105 critically ill patients in the report of Garonzik et $\mathrm{al}^{50} 16$ were receiving renal replacement therapy at the time of initiating the CMS regimen (12 intermittent hemodialysis and 4 continuous renal replacement). These renal replacement modalities were shown to have a substantial impact on the plasma colistin concentration achieved from a given daily dose of CMS; this was in agreement with reports from case studies and case series. ${ }^{54,61-66}$ There are two reasons why renal replacement therapy has such a substantial impact on dosage requirements of CMS. First, the circulating plasma concentrations of CMS are considerably higher than those of formed colistin (-Figs. $\mathbf{2}$ and $\mathbf{3}$ ) and therefore a significant proportion of the material dialyzed out of the patient is in the form of CMS, before there has been an opportunity for conversion to colistin in the body. Second, as noted above, colistin is subject to very extensive carrier-mediated tubular reabsorption in the kidney ${ }^{44}$ but a renal replacement cartridge has no corresponding mechanism to return to the circulation compounds, such as CMS and colistin, which have passively diffused into dialysate. As a result of the efficient extracorporeal clearance of CMS/colistin, dosage regimens of CMS for such patients must be carefully chosen. Garonzik et $\mathrm{al}^{50}$ by way of population PK modeling were able to propose a daily maintenance dose of CMS to achieve a desired steady-state plasma concentration of formed colistin in patients receiving intermittent hemodialysis. The algorithm that was developed for designing dosage regimens for patients on intermittent hemodialysis included 
administration of a supplemental dose of CMS after the dialysis session to replace CMS and colistin that had been cleared by dialysis. These authors also developed a CMS dosage algorithm to achieve a desired plasma concentration of formed colistin in patients receiving continuous renal replacement therapy. ${ }^{50}$

The study of Garonzik et $\mathrm{al}^{50}$ also developed an algorithm for calculating a loading dose of CMS to be administered to patients whether they are, or are not, receiving renal replacement therapy at the initiation of therapy. The loading dose algorithm was based upon body weight being a covariate on the volume of distribution of CMS. Alternatively, a nonweightbased loading dose may be used. ${ }^{67}$ The loading and maintenance doses proposed by Garonzik et al are the first scientifically based regimens for CMS/colistin. ${ }^{50}$ The study went on to recruit a total of 230 critically ill patients and therefore the interim dosing suggestions ${ }^{50}$ are not reproduced here as they will be updated based upon the final population PK/PD analysis of the data.

There is very little information on the extent to which colistin distributes into important extravascular infection sites (e.g., cerebrospinal fluid [CSF], lungs) following intravenous administration of CMS. Concentrations of formed colistin in CSF are very low compared with those in plasma. 53,68,69 In a similar way, following intravenously administered CMS the concentrations of formed colistin in sputum of patients with cystic fibrosis ${ }^{70}$ and in bronchoalveolar lavage (BAL) fluid from critically ill patients ${ }^{57}$ are very low relative to concomitant plasma concentrations. In relation to the latter study, it should be noted that BAL is an approximate 100 -fold dilution of epithelial lining fluid (ELF) and given the limit of quantification of the assay for colistin in BAL the result of that study requires cautious interpretation. However, the current data overall suggest limited penetration of formed colistin into CSF and lung fluids following intravenous administration of CMS.

\section{Administration of CMS Directly to the Central Nervous System and Lungs}

It is axiomatic that bacterial killing by an antibiotic at an extravascular infection site requires achievement of adequate concentrations of the antibiotic at that site. CMS is commonly administered to critically ill patients for the treatment of ventilator-associated pneumonia and less commonly for the treatment of infections within the central nervous system. However, as reviewed briefly in the last paragraph of the section above, the emerging data suggest that following intravenous administration of CMS the concentrations of formed colistin achieved in CSF and lung fluids are very low.

Two recent studies, the first in patients with cystic fibro$\operatorname{sis}^{70}$ and the second in mechanically ventilated critically ill patients, ${ }^{71}$ have demonstrated the substantially higher colistin concentrations that can be achieved in sputum and ELF, respectively, following inhalational delivery of CMS, compared with intravenous administration. Following pulmonary administration of CMS the extent of absorption into the systemic circulation was minimal and the plasma concentrations of formed colistin were very low. ${ }^{70,71}$ It was possible in the study in cystic fibrosis patients to calculate the pulmonary targeting advantage of inhalational administration (i.e., the relative values for inhalational versus intravenous administration of CMS of the ratio of colistin concentration in sputum to that in plasma). ${ }^{70}$ There was a massive targeting advantage with inhalational administration, indicating the potential to achieve more effective bacterial killing in the lungs while sparing the kidneys. The role of inhalational administration of CMS, possibly combined with a suitable intravenous regimen, in critically ill patients warrants further investigation.

Intrathecal or intraventricular administration of CMS appears to be a generally effective and safe treatment for ventriculitis/meningitis caused by gram-negative bacteria. ${ }^{72-75}$ A much lower dose is administered by these routes than is typically administered intravenously. Because of the relatively small volume into which the intrathecal or intraventricular dose is delivered and the relatively slow turnover of CSF, it is possible to achieve CSF colistin concentrations very much higher than is possible with intravenous administration of a far larger dose..$^{53,68,69}$ One would expect plasma colistin concentrations following intrathecal or intraventricular administration of CMS to be very low, although there appear to be no data to substantiate this. It can be noted, however, that colistin-associated nephrotoxicity appears to occur rarely following these routes of delivery to the CNS. ${ }^{72,74}$ There may be benefit in concomitant administration of intravenous CMS.

\section{Take-Home Messages}

The following are some key points for those using colistin in critically ill patients to keep in mind:

- How is colistin administered? Colistin is administered intravenously and by inhalation as its inactive prodrug CMS (also known as colistimethate). CMS must be converted to colistin in the body. Care is needed to avoid confusion arising from the different conventions used to label vials and specify doses.

- What plasma concentration is appropriate for intravenous administration? Based upon current evidence, a plasma colistin concentration of $2 \mathrm{mg} / \mathrm{L}$ is a reasonable target value for isolates with MICs $\leq 1 \mathrm{mg} / \mathrm{L}$, and minimizes the risk of nephrotoxicity.

- Should I consider colistin combination therapy? It is prudent to consider combination therapy for infections where the causative organism has an MIC $>1 \mathrm{mg} / \mathrm{L}$ or when there is a high-inoculum or deep-seated infection (e.g., in lungs), especially in patients with moderate-to-good renal function, although the clinical benefit of colistin combinations remains unproven.

- Do I need to administer an intravenous loading dose? Yes, because CMS is relatively slowly converted to colistin in the body and it may take many hours to achieve steady-state plasma concentrations without a loading dose.

- Do I need to adjust the daily maintenance dose if the patient has renal impairment? The plasma concentrations of 
colistin achieved from a given intravenous daily dose are influenced by kidney function. The recently developed dosing algorithm provides a means to tailor the daily dose.

- Does renal replacement therapy have implications for selection of intravenous dosage regimens? Yes, CMS and colistin are efficiently removed from the body by both intermittent hemodialysis and continuous renal replacement therapy. The recently developed dosage algorithms for such patients allow calculation of dosage regimens and of the size of a supplemental dose to be administered after each intermittent hemodialysis session.

- Is there a potential benefit of using TDM to assist optimizing therapy? Yes, colistin has a narrow therapeutic window and plasma concentrations are subject to marked interpatient variability, even at a given creatinine clearance and daily dose of intravenous CMS. TDM is recommended if it is available.

- Should I consider administration directly to the lungs or CNS for infections in those sites? Intrathecal or intraventricular administration of CMS is able to generate concentrations of colistin in CSF that are very much higher than can be achieved with intravenous administration, and the treatment appears to be safe and effective. Similarly, inhalational delivery of CMS generates concentrations of colistin in lung fluids that are substantially higher than is possible after intravenous administration, with negligible plasma concentrations. The potential benefits (more effective bacterial killing in lung and sparing of the kidneys) are attractive, but remain to be proven.

In summary, over the last decade or so, considerable progress has been made in understanding how to optimize the clinical use of colistin in critically ill patients. However, as identified in this article, answers are still required for several important questions.

\section{Acknowledgment}

This work was supported by the Australian National Health and Medical Research Council (NHMRC, Career Development fellowship number 1062509 to C. B. L.).

\section{References}

1 Koch-Weser J, Sidel VW, Federman EB, Kanarek P, Finer DC, Eaton AE. Adverse effects of sodium colistimethate. Manifestations and specific reaction rates during 317 courses of therapy. Ann Intern Med 1970;72(6):857-868

2 Li J, Nation RL, Turnidge JD, et al. Colistin: the re-emerging antibiotic for multidrug-resistant Gram-negative bacterial infections. Lancet Infect Dis 2006;6(9):589-601

3 Bergen PJ, Li J, Nation RL. Dosing of colistin-back to basic PK/PD. Curr Opin Pharmacol 2011;11(5):464-469

4 Hede K. Antibiotic resistance: An infectious arms race. Nature 2014;509(7498):S2-S3

5 Roberts JA, Abdul-Aziz MH, Lipman J, et al; International Society of Anti-Infective Pharmacology and the Pharmacokinetics and Pharmacodynamics Study Group of the European Society of Clinical Microbiology and Infectious Diseases. Individualised antibiotic dosing for patients who are critically ill: challenges and potential solutions. Lancet Infect Dis 2014;14(6):498-509

6 Bergen PJ, Landersdorfer CB, Lee HJ, Li J, Nation RL. 'Old' antibiotics for emerging multidrug-resistant bacteria. Curr Opin Infect Dis 2012;25(6):626-633

7 Bergen PJ, Landersdorfer CB, Zhang J, et al. Pharmacokinetics and pharmacodynamics of 'old' polymyxins: what is new? Diagn Microbiol Infect Dis 2012;74(3):213-223

8 Li J, Nation RL, Milne RW, Turnidge JD, Coulthard K. Evaluation of colistin as an agent against multi-resistant Gram-negative bacteria. Int J Antimicrob Agents 2005;25(1):11-25

9 Bergen PJ, Li J, Rayner CR, Nation RL. Colistin methanesulfonate is an inactive prodrug of colistin against Pseudomonas aeruginosa. Antimicrob Agents Chemother 2006;50(6):1953-1958

10 Barnett M, Bushby SR, Wilkinson S. Sodium sulphomethyl derivatives of polymyxins. Br Pharmacol Chemother 1964;23:552-574

11 Li J, Milne RW, Nation RL, Turnidge JD, Coulthard K. Stability of colistin and colistin methanesulfonate in aqueous media and plasma as determined by high-performance liquid chromatography. Antimicrob Agents Chemother 2003;47(4):1364-1370

12 Dudhani RV, Nation RL, Li J. Evaluating the stability of colistin and colistin methanesulphonate in human plasma under different conditions of storage. J Antimicrob Chemother 2010;65(7): 1412-1415

13 Nation RL, Li J, Cars O, et al. Consistent global approach on reporting of colistin doses to promote safe and effective use. Clin Infect Dis 2014;58(1):139-141

14 Institute for Safe Medication Practices (ISMP). National alert issued: Dosing confusion with colistimethate for injection, 2011. Available at: http://www.ismp.org/pressroom/PR20110629.pdf. Accessed 24 August, 2014

15 Gales AC, Jones RN, Sader HS. Contemporary activity of colistin and polymyxin B against a worldwide collection of Gram-negative pathogens: results from the SENTRY Antimicrobial Surveillance Program (2006-09). J Antimicrob Chemother 2011;66(9): 2070-2074

16 Gales AC, Castanheira M, Jones RN, Sader HS. Antimicrobial resistance among Gram-negative bacilli isolated from Latin America: results from SENTRY Antimicrobial Surveillance Program (Latin America, 2008-2010). Diagn Microbiol Infect Dis 2012;73(4): 354-360

17 Hawser SP. Susceptibility of Klebsiella pneumoniae clinical isolates from 2007 to 2009 to colistin and comparator antibiotics. Int J Antimicrob Agents 2010;36(4):383-384

18 Nakamura T, Komatsu M, Yamasaki K, et al. Susceptibility of various oral antibacterial agents against extended spectrum $\beta$-lactamase producing Escherichia coli and Klebsiella pneumoniae. J Infect Chemother 2014;20(1):48-51

19 The European Committee on Antimicrobial Susceptibility Testing. Breakpoint tables for interpretation of MICs and zone diameters. Version 4.0 2014. Available at: http://www.eucast.org. Accessed 24 August, 2014

20 Clinical and Laboratory Standards Institute (CLSI). Performance standards for antimicrobial susceptibility testing; twenty-third informational supplement. 2013

21 Velkov T, Thompson PE, Nation RL, Li J. Structure-activity relationships of polymyxin antibiotics. J Med Chem 2010;53(5): 1898-1916

22 Hancock RE. Alterations in outer membrane permeability. Annu Rev Microbiol 1984;38:237-264

23 Moffatt JH, Harper M, Harrison P, et al. Colistin resistance in Acinetobacter baumannii is mediated by complete loss of lipopolysaccharide production. Antimicrob Agents Chemother 2010; 54(12):4971-4977

24 Li J, Turnidge J, Milne R, Nation RL, Coulthard K. In vitro pharmacodynamic properties of colistin and colistin methanesulfonate against Pseudomonas aeruginosa isolates from patients with 
cystic fibrosis. Antimicrob Agents Chemother 2001;45(3): 781-785

25 Bergen PJ, Li J, Nation RL, Turnidge JD, Coulthard K, Milne RW. Comparison of once-, twice- and thrice-daily dosing of colistin on antibacterial effect and emergence of resistance: studies with Pseudomonas aeruginosa in an in vitro pharmacodynamic model. J Antimicrob Chemother 2008;61(3):636-642

26 Bergen PJ, Bulitta JB, Forrest A, Tsuji BT, Li J, Nation RL. Pharmacokinetic/pharmacodynamic investigation of colistin against Pseudomonas aeruginosa using an in vitro model. Antimicrob Agents Chemother 2010;54(9):3783-3789

27 Bergen PJ, Tsuji BT, Bulitta JB, et al. Synergistic killing of multidrugresistant Pseudomonas aeruginosa at multiple inocula by colistin combined with doripenem in an in vitro pharmacokinetic/pharmacodynamic model. Antimicrob Agents Chemother 2011; 55(12):5685-5695

28 Lora-Tamayo J, Murillo O, Bergen PJ, et al. Activity of colistin combined with doripenem at clinically relevant concentrations against multidrug-resistant Pseudomonas aeruginosa in an in vitro dynamic biofilm model. J Antimicrob Chemother 2014; 69(9):2434-2442

29 Poudyal A, Howden BP, Bell JM, et al. In vitro pharmacodynamics of colistin against multidrug-resistant Klebsiella pneumoniae. J Antimicrob Chemother 2008;62(6):1311-1318

30 Deris ZZ, Yu HH, Davis K, et al. The combination of colistin and doripenem is synergistic against Klebsiella pneumoniae at multiple inocula and suppresses colistin resistance in an in vitro pharmacokinetic/pharmacodynamic model. Antimicrob Agents Chemother 2012;56(10):5103-5112

31 Li J, Rayner CR, Nation RL, et al. Heteroresistance to colistin in multidrug-resistant Acinetobacter baumannii. Antimicrob Agents Chemother 2006;50(9):2946-2950

32 Tan CH, Li J, Nation RL. Activity of colistin against heteroresistant Acinetobacter baumannii and emergence of resistance in an in vitro pharmacokinetic/pharmacodynamic model. Antimicrob Agents Chemother 2007;51(9):3413-3415

33 Owen RJ, Li J, Nation RL, Spelman D. In vitro pharmacodynamics of colistin against Acinetobacter baumannii clinical isolates. J Antimicrob Chemother 2007;59(3):473-477

34 Lee HJ, Bergen PJ, Bulitta JB, et al. Synergistic activity of colistin and rifampin combination against multidrug-resistant Acinetobacter baumannii in an in vitro pharmacokinetic/pharmacodynamic model. Antimicrob Agents Chemother 2013;57(8):3738-3745

35 Bulitta JB, Yang JC, Yohonn L, et al. Attenuation of colistin bactericidal activity by high inoculum of Pseudomonas aeruginosa characterized by a new mechanism-based population pharmacodynamic model. Antimicrob Agents Chemother 2010;54(5): 2051-2062

36 Forrest A, Silveira FP, Thamlikitkul V, et al. Risk factors for colistinassociated nephrotoxicity. In: Proceedings of the 1st International Conference on Polymyxins; May 2-4, 2013; Prato, Italy

37 Sorlí L, Luque S, Grau S, et al. Trough colistin plasma level is an independent risk factor for nephrotoxicity: a prospective observational cohort study. BMC Infect Dis 2013;13:380

38 Dudhani RV, Turnidge JD, Coulthard K, et al. Elucidation of the pharmacokinetic/pharmacodynamic determinant of colistin activity against Pseudomonas aeruginosa in murine thigh and lung infection models. Antimicrob Agents Chemother 2010; 54(3):1117-1124

39 Dudhani RV, Turnidge JD, Nation RL, Li J. fAUC/MIC is the most predictive pharmacokinetic/pharmacodynamic index of colistin against Acinetobacter baumannii in murine thigh and lung infection models. J Antimicrob Chemother 2010;65(9):1984-1990

40 Bergen PJ, Forrest A, Bulitta JB, et al. Clinically relevant plasma concentrations of colistin in combination with imipenem enhance pharmacodynamic activity against multidrug-resistant Pseudomonas aeruginosa at multiple inocula. Antimicrob Agents Chemother 2011;55(11):5134-5142
41 Clancy CJ, Chen L, Hong JH, et al. Mutations of the ompK36 porin gene and promoter impact responses of sequence type 258, KPC-2producing Klebsiella pneumoniae strains to doripenem and doripenem-colistin. Antimicrob Agents Chemother 2013;57(11): 5258-5265

42 Zusman O, Avni T, Leibovici L, et al. Systematic review and metaanalysis of in vitro synergy of polymyxins and carbapenems. Antimicrob Agents Chemother 2013;57(10):5104-5111

43 Paul M, Carmeli Y, Durante-Mangoni E, et al. Combination therapy for carbapenem-resistant Gram-negative bacteria. J Antimicrob Chemother 2014;69(9):2305-2309

44 Li J, Milne RW, Nation RL, Turnidge JD, Smeaton TC, Coulthard K. Use of high-performance liquid chromatography to study the pharmacokinetics of colistin sulfate in rats following intravenous administration. Antimicrob Agents Chemother 2003;47(5): 1766-1770

45 Li J, Milne RW, Nation RL, Turnidge JD, Smeaton TC, Coulthard K. Pharmacokinetics of colistin methanesulphonate and colistin in rats following an intravenous dose of colistin methanesulphonate. J Antimicrob Chemother 2004;53(5):837-840

46 Marchand S, Lamarche I, Gobin P, Couet W. Dose-ranging pharmacokinetics of colistin methanesulphonate (CMS) and colistin in rats following single intravenous CMS doses. J Antimicrob Chemother 2010;65(8):1753-1758

$47 \mathrm{Li} \mathrm{J}$, Coulthard K, Milne R, et al. Steady-state pharmacokinetics of intravenous colistin methanesulphonate in patients with cystic fibrosis. J Antimicrob Chemother 2003;52(6):987-992

48 Plachouras D, Karvanen M, Friberg LE, et al. Population pharmacokinetic analysis of colistin methanesulfonate and colistin after intravenous administration in critically ill patients with infections caused by gram-negative bacteria. Antimicrob Agents Chemother 2009;53(8):3430-3436

49 Couet W, Grégoire N, Gobin P, et al. Pharmacokinetics of colistin and colistimethate sodium after a single $80-\mathrm{mg}$ intravenous dose of CMS in young healthy volunteers. Clin Pharmacol Ther 2011; 89(6):875-879

50 Garonzik SM, Li J, Thamlikitkul V, et al. Population pharmacokinetics of colistin methanesulfonate and formed colistin in critically ill patients from a multicenter study provide dosing suggestions for various categories of patients. Antimicrob Agents Chemother 2011;55(7):3284-3294

51 Theuretzbacher U. Product information for parenteral colistin varies substantially across Europe. J Antimicrob Chemother 2014;69(7):1987-1992

52 Nation RL, Velkov T, Li J. Colistin and Polymyxin B: Peas in a Pod, or Chalk and Cheese? Clin Infect Dis 2014

53 Antachopoulos C, Karvanen M, Iosifidis E, et al. Serum and cerebrospinal fluid levels of colistin in pediatric patients. Antimicrob Agents Chemother 2010;54(9):3985-3987

54 Li J, Rayner CR, Nation RL, et al. Pharmacokinetics of colistin methanesulfonate and colistin in a critically ill patient receiving continuous venovenous hemodiafiltration. Antimicrob Agents Chemother 2005;49(11):4814-4815

55 Trotman RL, Williamson JC, Shoemaker DM, Salzer WL. Antibiotic dosing in critically ill adult patients receiving continuous renal replacement therapy. Clin Infect Dis 2005;41(8):1159-1166

56 Markou N, Markantonis SL, Dimitrakis E, et al. Colistin serum concentrations after intravenous administration in critically ill patients with serious multidrug-resistant, gram-negative bacilli infections: a prospective, open-label, uncontrolled study. Clin Ther 2008;30(1):143-151

57 Imberti R, Cusato M, Villani P, et al. Steady-state pharmacokinetics and BAL concentration of colistin in critically Ill patients after IV colistin methanesulfonate administration. Chest 2010;138(6): 1333-1339

58 Mohamed AF, Karaiskos I, Plachouras D, et al. Application of a loading dose of colistin methanesulfonate in critically ill patients: population pharmacokinetics, protein binding, and prediction of 
bacterial kill. Antimicrob Agents Chemother 2012;56(8): 4241-4249

59 Kumar A, Roberts D, Wood KE, et al. Duration of hypotension before initiation of effective antimicrobial therapy is the critical determinant of survival in human septic shock. Crit Care Med 2006;34(6):1589-1596

60 Luna CM, Aruj P, Niederman MS, et al; Grupo Argentino de Estudio de la Neumonía Asociada al Respirador group. Appropriateness and delay to initiate therapy in ventilator-associated pneumonia. Eur Respir J 2006;27(1):158-164

61 Karvanen M, Plachouras D, Friberg LE, et al. Colistin methanesulfonate and colistin pharmacokinetics in critically ill patients receiving continuous venovenous hemodiafiltration. Antimicrob Agents Chemother 2013;57(1):668-671

62 Luque S, Sorli L, Li J, et al. Effective removal of colistin methanesulphonate and formed colistin during intermittent haemodialysis in a patient infected by polymyxin-only-susceptible Pseudomonas aeruginosa. J Chemother 2014;26(2):122-124

63 Markou N, Fousteri M, Markantonis SL, et al. Colistin pharmacokinetics in intensive care unit patients on continuous venovenous haemodiafiltration: an observational study. J Antimicrob Chemother 2012;67(10):2459-2462

64 Marchand S, Frat JP, Petitpas F, et al. Removal of colistin during intermittent haemodialysis in two critically ill patients. J Antimicrob Chemother 2010;65(8):1836-1837

65 Strunk AK, Schmidt JJ, Baroke E, et al. Single- and multiple-dose pharmacokinetics and total removal of colistin in a patient with acute kidney injury undergoing extended daily dialysis. J Antimicrob Chemother 2014;69(7):2008-2010

66 Leporati M, Bua RO, Mariano F, et al. Determination by LC-MS/MS of colistins A and B in plasma and ultrafiltrate from critically ill patients undergoing continuous venovenous hemodiafiltration. Ther Drug Monit 2014;36(2):182-191

67 Mohamed AF, Cars O, Friberg LE. A pharmacokinetic/pharmacodynamic model developed for the effect of colistin on Pseudomonas aeruginosa in vitro with evaluation of population pharmacokinetic variability on simulated bacterial killing. J Antimicrob Chemother 2014;69(5):1350-1361

68 Markantonis SL, Markou N, Fousteri M, et al. Penetration of colistin into cerebrospinal fluid. Antimicrob Agents Chemother 2009; 53(11):4907-4910

69 Ziaka M, Markantonis SL, Fousteri M, et al. Combined intravenous and intraventricular administration of colistin methanesulfonate in critically ill patients with central nervous system infection. Antimicrob Agents Chemother 2013;57(4):1938-1940

70 W S Yapa S, Li J, Patel K, et al. Pulmonary and systemic pharmacokinetics of inhaled and intravenous colistin methanesulfonate in cystic fibrosis patients: targeting advantage of inhalational administration. Antimicrob Agents Chemother 2014;58(5): 2570-2579

71 Athanassa ZE, Markantonis SL, Fousteri MZ, et al. Pharmacokinetics of inhaled colistimethate sodium (CMS) in mechanically ventilated critically ill patients. Intensive Care Med 2012;38(11): 1779-1786

72 Bargiacchi O, Rossati A, Car P, et al. Intrathecal/intraventricular colistin in external ventricular device-related infections by multidrug resistant Gram negative bacteria: case reports and review. Infection 2014;42(5):801-809

73 Imberti R, Cusato M, Accetta G, et al. Pharmacokinetics of colistin in cerebrospinal fluid after intraventricular administration of colistin methanesulfonate. Antimicrob Agents Chemother 2012; 56(8):4416-4421

74 Karaiskos I, Galani L, Baziaka F, Giamarellou H. Intraventricular and intrathecal colistin as the last therapeutic resort for the treatment of multidrug-resistant and extensively drug-resistant Acinetobacter baumannii ventriculitis and meningitis: a literature review. Int J Antimicrob Agents 2013;41(6):499-508

75 Karaiskos I, Galani L, Baziaka F, et al. Successful treatment of extensively drug-resistant Acinetobacter baumannii ventriculitis and meningitis with intraventricular colistin after application of a loading dose: a case series. Int J Antimicrob Agents 2013;41(5): 480-483 